\title{
In silico structural homology modelling of EST073 motif coding protein of tea Camellia sinensis $(\mathrm{L})$
}

\author{
K. H. T. Karunarathna ${ }^{1,2^{*}}$ (D, N. H. K. S. Senathilake ${ }^{1}$, K. M. Mewann ${ }^{3}$, O. V. D. S. J. Weerasena ${ }^{1}$ and S. A. C. N. Perera ${ }^{4}$
}

\begin{abstract}
Background: Tea (Camellia sinensis (L). O. Kuntze) is known as the oldest, mild stimulating caffeine containing nonalcoholic beverage. One of the major threats in south Asian tea industry is the blister blight leaf disease (BB), caused by the fungus Exobasidium vexans Masse. SSR DNA marker EST SSR 073 is used as a molecular marker to tag blister blight disease resistance trait of tea. The amino acid sequences were derived from CDNA sequences related to EST SSR 073 of BB susceptible (TRI 2023) and BB resistant (TRI 2043) cultivars. An attempt has been made to understand the structural characteristics and variations of EST SSR 073 locus that may reveal the factors influencing the BB resistance of tea with multiple bioinformatics tools such as ORF finder, ExPasy ProtParam tools, modeler V 9.17, Rampage server, UCSF-Chimera, and HADDOCK docking server.

Results: The primary, secondary, and tertiary structures of EST SSR 073 coding protein were analyzed using the amino acid sequences of both BB resistant TRI 2043 and BB susceptible TRI 2023 tea cultivars. The coding amino acid sequences of both the cultivars were homologous to photosystem I subunit protein (PsaD I) of Pisum sativum. The predicted 3D structures of proteins were validated and considered as an acceptable overall stereochemical quality. The BB resistant protein showed $\mathrm{CT}$ repeat extension and did not involve in topology of the PsaD I subunit. The $\mathrm{C}$ terminal truncation of $\mathrm{BB}$ resistance caused the formation of hydrogen bonds interacting with PsaD I and other subunits of photosystem I in the modeled three-dimensional protein structure.

Conclusions: Camellia sinensis EST 073 SSR motif coding protein was identified as the PsaD I subunit of photosystem I. The exact mechanism of PsaD I conferring the resistance for blister blight in tea needs to be further investigated.
\end{abstract}

Keywords: Blister blight disease, EST-SSR, Homology modeling, Molecular markers, Tea

\section{Background}

Tea, Camellia sinensis (L.) O. Kuntze, is the second most popular, healthy non-alcoholic beverage in the world. It is an economically important tree crop, grown in several countries in Asia and Africa. Globally, Sri Lanka is the third-largest producer and the $2^{\text {nd }}$ largest

\footnotetext{
* Correspondence: thissa1234@gmail.com

'Institute of Biochemistry, Molecular Biology and Biotechnology, University of

Colombo, Colombo, Sri Lanka

${ }^{2}$ Current address: Department of biosystems Technology, Faculty of

Technology, University of Ruhuna, Matara, Sri Lanka

Full list of author information is available at the end of the article
}

exporter of tea [1] with its popular brand "Ceylon Tea", playing a key role in the international tea trade.

Blister blight leaf disease (BB), caused by the obligatory fungal pathogen, Exobasidium vexans Masse (Basidiomycetes) is one of the most devastating biotic constraints, commonly found in a majority of tea plantations in south Asia including Sri Lanka, India, Indonesia, Bangladesh, Thailand, Nepal, Vietnam, Cambodia, and Japan [2]. The BB leaf disease causes approximately 25 to $30 \%$ crop loss annually depending on the agro-ecological region (AER) of Sri Lanka [3]. The disease infection also causes a reduction of the quality of black tea by changing the composition of leaf 
biochemical components such as polyphenols, catechins, and enzymes which highly influence the quality of black tea [4].

Presently, the control of this disease is solely based on chemical means, where spraying of $\mathrm{Cu}$-based fungicides directly on to the foliage, before infection, being the recommended practice. The disease is very common in major tea growing areas of Sri Lanka throughout the year, and the repeated application of $\mathrm{Cu}$-based fungicides may lead to chemical residues in the end product "Black tea." Though tea, is a popular healthy beverage, exceeding maximum residual levels (MRLs) of pesticides, heavy metals, and other chemical impurities, leads to a non-tariff trade barrier in exporting and consumption of tea [5]. Therefore, to overcome the said constraints and also to maintain the quality of the symbol "Ceylon Tea", the development of resistant cultivars to $\mathrm{BB}$ disease would be the most effective and sustainable approach to control the disease.

Tea is a perennial crop, which requires $20-25$ years to develop a new improved cultivar and therefore, the application of marker-assisted selection (MAS) techniques would be highly desirable to increase the efficiency and effectiveness of the breeding program. Bulk segregant analysis (BSA) approach has successfully been applied to identify a SSR DNA marker EST SSR 073 to tag blister blight disease resistance trait using a segregating population derived from the two parents: TRI 2043 (resistant cultivar) $\times$ TRI 2023 (susceptible cultivar) [6]. EST SSR 073 motif correlates with the photosystem I subunit D (PsaD I) and identifying the structural model of a protein of the motif is one of the key points for understanding the underlying biological mechanism at a molecular level. The available knowledge on the structure and the role of PsaD I protein is scarce. The experimental elucidation of the tertiary structure of a protein is a huge and a difficult endeavor [7]. The X-ray crystallography or nuclear magnetic resonance techniques (NMR), which are applied to identify the tertiary structure of a protein, are time consuming and expensive $[8,9]$. However, the "In silico homology modeling" provides an alternative application to predict the 3D structure of proteins with better validation. Homology modeling is known to be one of the best and extensively used computational methods to generate three-dimensional structures when there is more than $35 \%$ sequence identity between the known protein structure (template) and the unknown protein structure [10-13].

In silico homology modeling has been successfully applied to predict the structure of Matrix metalloproteinase 25 (MMP 25) and it can be used as a target for the inhibition of airway remodeling in asthma disease by using in silico drug designing methods [14]. Furthermore, an acceptable protein structure of nif A which is involved in nitrogen fixation of rhizobial strains, has been identified and validated by using in silico structure homology modeling [15]. In silico characterization of ChiLCV coat proteins of Begomovirus in chilli aided in the development of strategies to control Begomovirus disease of crops [16]. Vascular wilt disease of tomato caused by Fusarium oxysporum $f$. sp. lycopersici is controlled by targeting a novel candidate protein FOXG_04696 which has been developed by homology modeling [17].

With the above background, molecular modeling of EST SSR 073 motif coding protein was the objective of the current study to provide a topology for revealing protein folding and functional structure which would help in understanding the blister blight fungal infection for combating the disease.

\section{Methods}

\section{DNA sequence of EST SSR 073 motif}

The EST SSR 073 motif containing cDNA sequence of blister blight disease resistant tea cultivar TRI 2043 (BBR) (GenBank accession no: MT303817) [18] and the DNA sequence of EST SSR 073 motif of blister blight disease susceptible tea cultivar TRI 2023 (BBS) (GenBank accession no: MT303818) [6] were retrieved. The sequences of BBR and BBS were aligned with BLASTn program [19].

\section{Amino acid sequence analysis and template retrieval}

All possible open reading frames (ORFs) for both the nucleotide sequences were identified by ORF finder (NCBI) [20]. Amino acid sequences derived by conceptual translation of each of the ORFs were used as the query for searching homologous sequences using BLASTp [21] against uniprotKB/swissprot database to identify potential orthologs [22]. The search was repeated against Protein Data Bank (PDB) and the amino acid sequence which contained the putative conserved domain and showed the highest sequence similarity and the lowest $E$ value, was selected for structure modeling.

\section{Homology modeling and energy minimization}

PsaD subunits have been reported to possess N (1Met to GLY 90) and C (Asp171 to Gly 193) terminal unstructured domains which are involved in the assembly of photosystem I super complex [23, 24]. Accordingly, the three-dimensional (3D) structure of the identified proteins were built using modeler V 9.17 [25] using the crystal structure of PsaD subunit of Pisum sativum photosystem I super-complex (PDB ID: 518r_D) [26] as the template and viewed by UCSF Chimera [27]. The generated model of $C$. sinensis PsaD-like protein was superimposed on the PasD subunit of $518 \mathrm{r}$ in energy minimized state while keeping the rest of the complex fixed. Superimposition was carried out using Matchmaker function of UCSF-Chimera [28, 29]. Energy minimization 


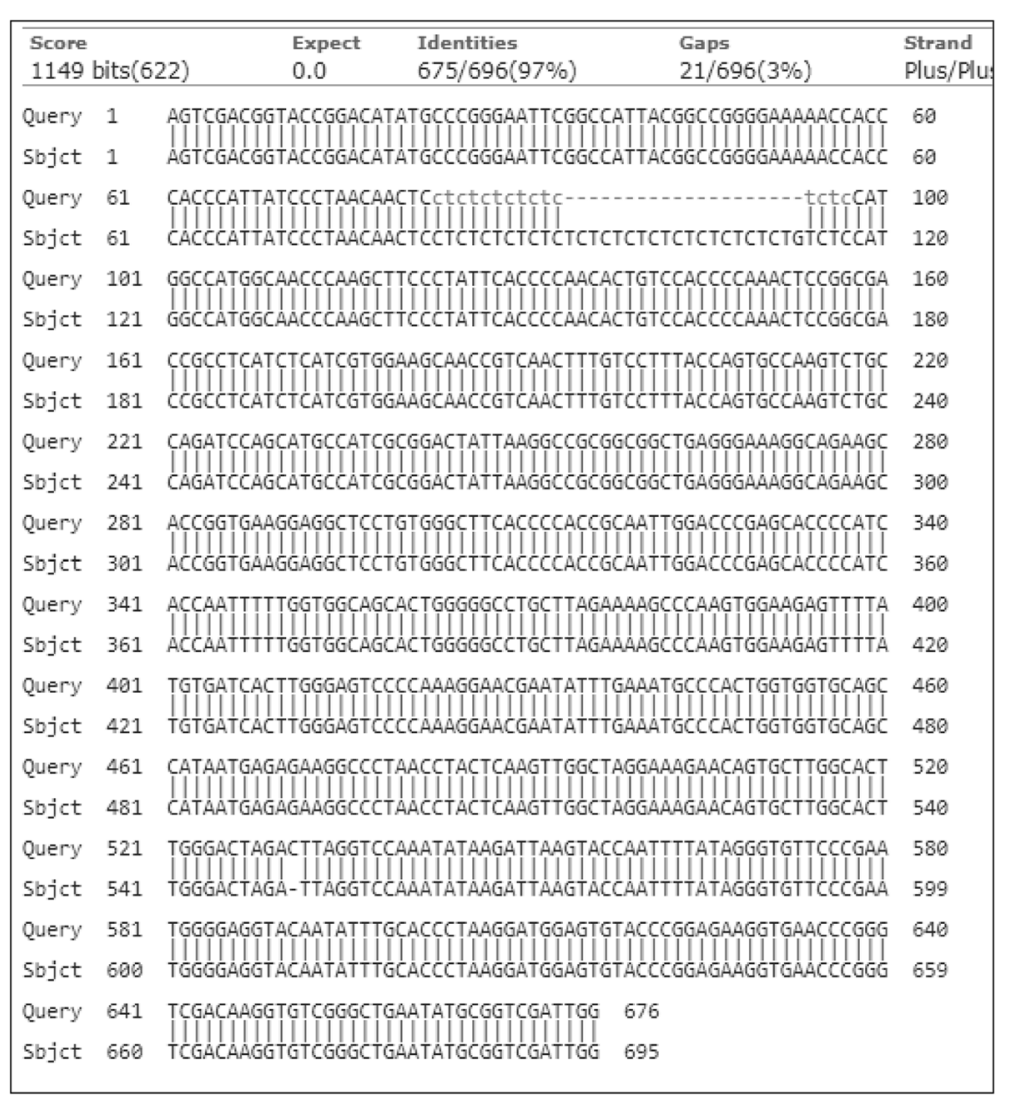

Fig. 1 Global pairwise alignment of EST 073 loci related to BB resistant and BB susceptible nucleotide sequences. Query denotes the BBS sequence and sbjct denotes BBR sequences.

was carried out using AMBER force field [30-32] in chimera with 100 steps of steepest descents followed by 10 steps of conjugate gradients.

\section{Protein model validation}

The quality of generated models was validated with respect to backbone and side chain geometry. To validate protein backbone quality, Ramachandran plot [33] was generated using Rampage server (http://mordred.bioc. cam.ac.uk/ rapper/rampage.php) and the backbone quality was validated by analyzing $\phi$ and $\psi$ angles using Ramachandran plot. Further; VERIFY3D, ERRAT,
PROVE, PROCHECK, AND WHATCHECK [34] servers were used to analyze the overall quality of the model.

\section{Structural comparison of modeled proteins}

Optimized energy minimized protein models generated for the sequence derived from BB susceptible TRI 2023 and the sequence derived from BB resistant TRI 2043 were superimposed using Matchmaker function of UCSF Chimera and RMSD (root mean square deviation) value was obtained. Further, structural comparison was carried out by superimposing and RMSD evaluation against the template protein that was used to generate the protein models.

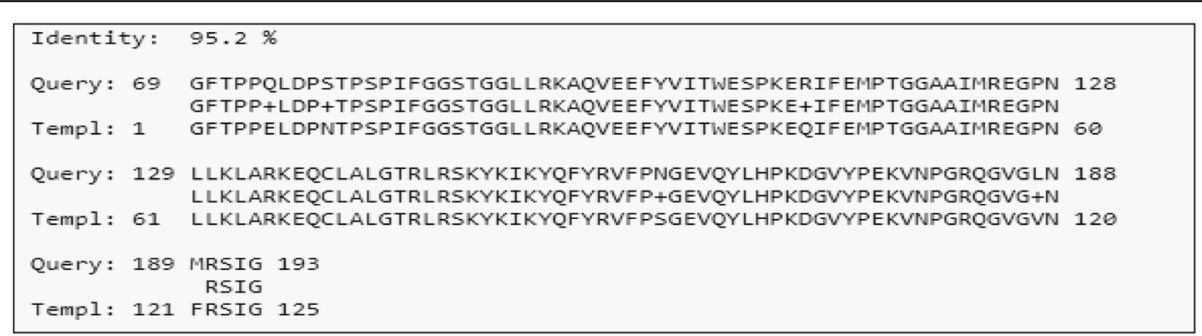

Fig. 2 Protein sequence alignment of BBS and P. sativum 


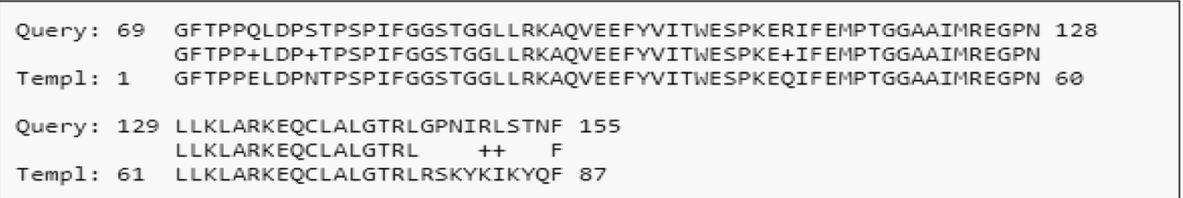

Fig. 3 Protein sequence alignment of BBR and P. sativum

\section{Validation of physiological parameters}

Structure-function relationship of the derived protein models was further validated using ProtParam tool of ExPASy Proteomics Server [35] for various parameters such as estimated half-life, theoretical pI, instability index, aliphatic index, and grand average of hydropathicity (GRAVY). The values were compared with the protein sequence used as the template as well as the BB susceptible and resistant genotypes.

\section{Molecular docking}

Crystal structure of PSI-I complex of Pisum sativum (PDB ID: 5l8r) was retrieved from RCSB-PDB. Subunits that interact with PsaD were retained and other subunits were removed using UCSF-Chimera. Modeled PsaD was docked against the binding site of the complex by HADDOCK docking server [36]. Docking results were viewed using UCSF Chimera. Default parameters were used for docking process and energy (E) values of each docking event were obtained. For comparative analysis, docked complexes were compared with the interactions of the PSI complex of Pisum sativum.

\section{Results}

Nucleotide sequence analysis and comparison

The best aligning nucleotide sequence of the Genbank database for both sequences was Camellia sinensis photosystem I reaction center subunit II, chloroplasticlike (LOC114287061), and mRNA (sequence ID: XM_02 8230326.1) derived from shuchazao tea cultivar of China. The EST SSR 073 motif containing cDNA sequence of blister blight disease resistant tea cultivar TRI 2043 displayed $99.07 \%$ identity with $0.0 \% E$ value by covering $92 \%$ query coverage. The cDNA sequence of blister blight disease susceptible tea cultivar TRI 2023 showed 99.33\% identity with $0.0 \% E$ value by covering $92 \%$ query coverage. Further, nucleotide sequences obtained for both BBS and BBR had over $80 \%$ identity with the coding sequence of Diospyros kaki photosystem I subunit D-I (PsaD-1) mRNA (ID: KX871204.1) with $E$ value of $5 \mathrm{e}-117$.

Pairwise alignment of BBS and BBR sequences indicated 20 microsatellite CT repeat extension in 5'UTR of $\mathrm{BBR}$ and a single nucleotide deletion at $552 \mathrm{bp}$ (deletion of C) (Fig. 1).

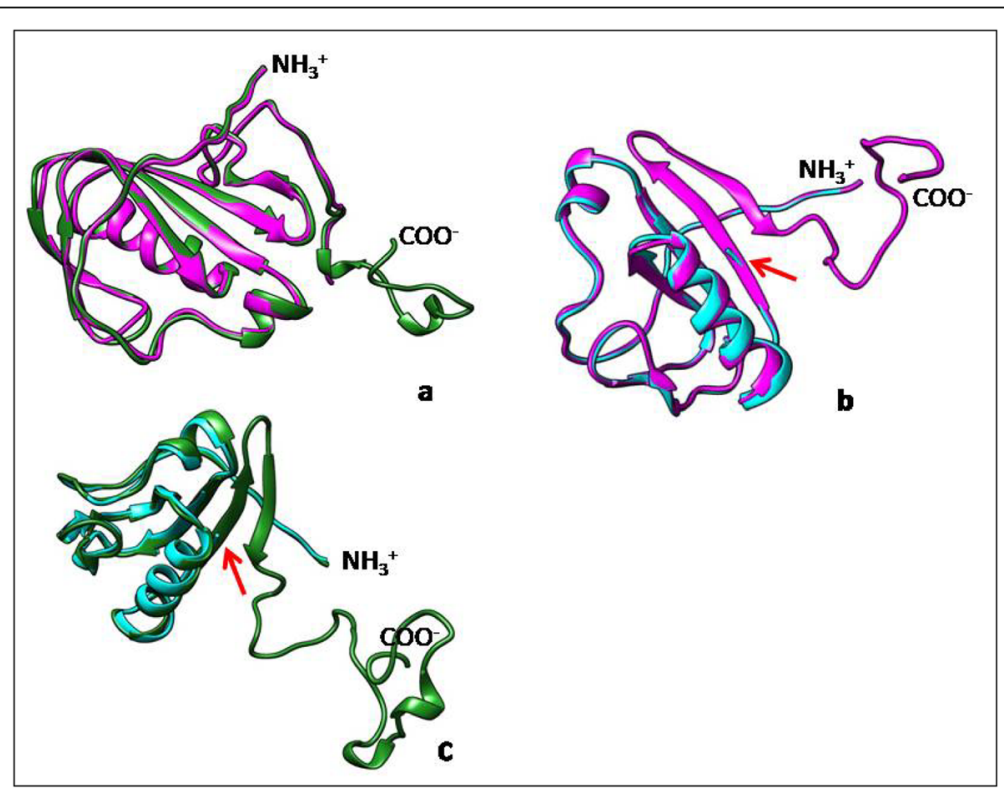

Fig. 4 Superposition of the generated models with the PsaD subunit of $P$. sativam (red colored arrows indicate the truncation of PsaD subunit of BBR). a- PsaD subunits from BBS (magenta) and P. sativum (green). b- PsaD subunits from BBR (cyan) and BBS (magenta). c- PsaD subunits from BBR (cyan) P. sativum (green) 

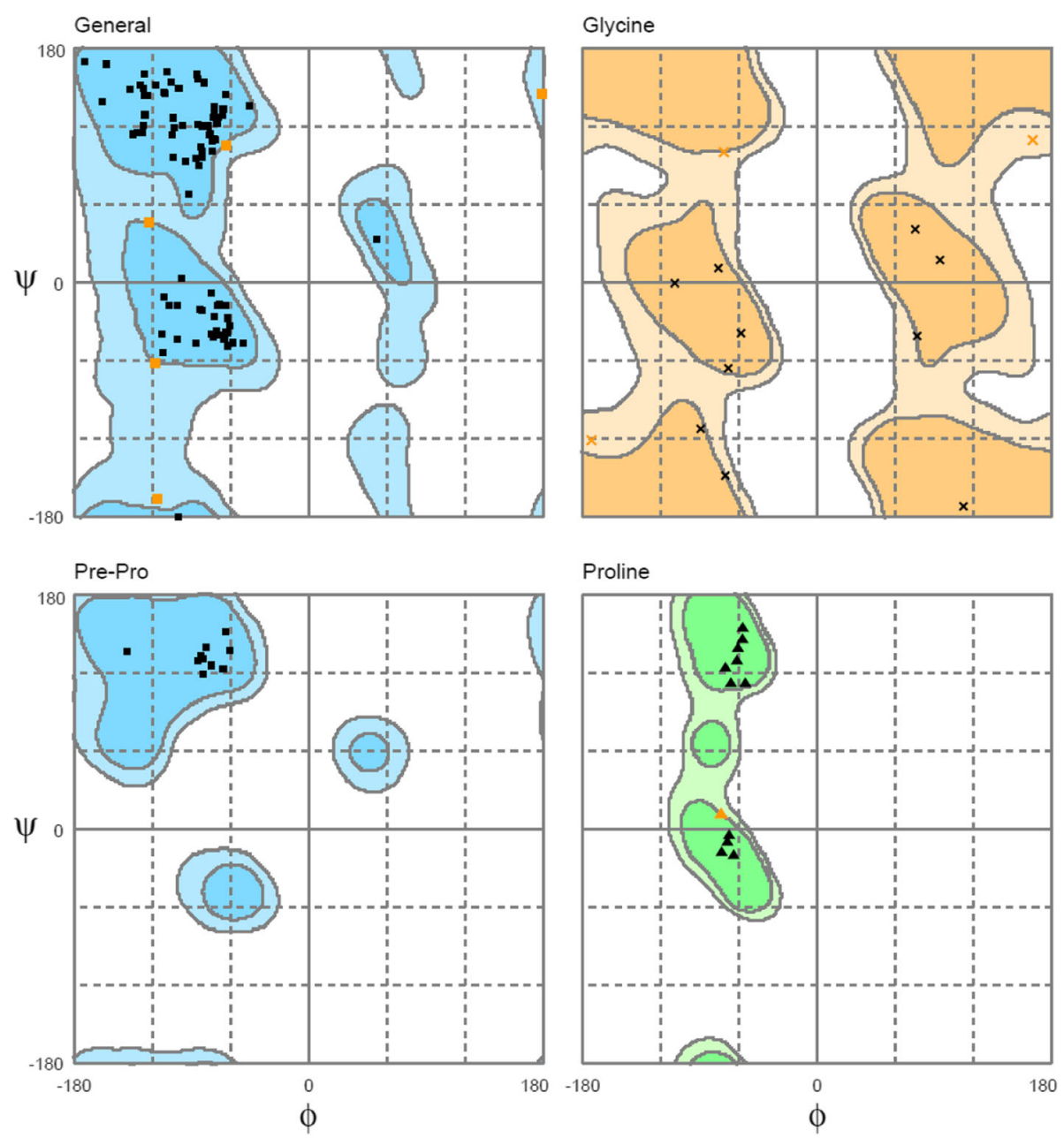

\section{Proline}
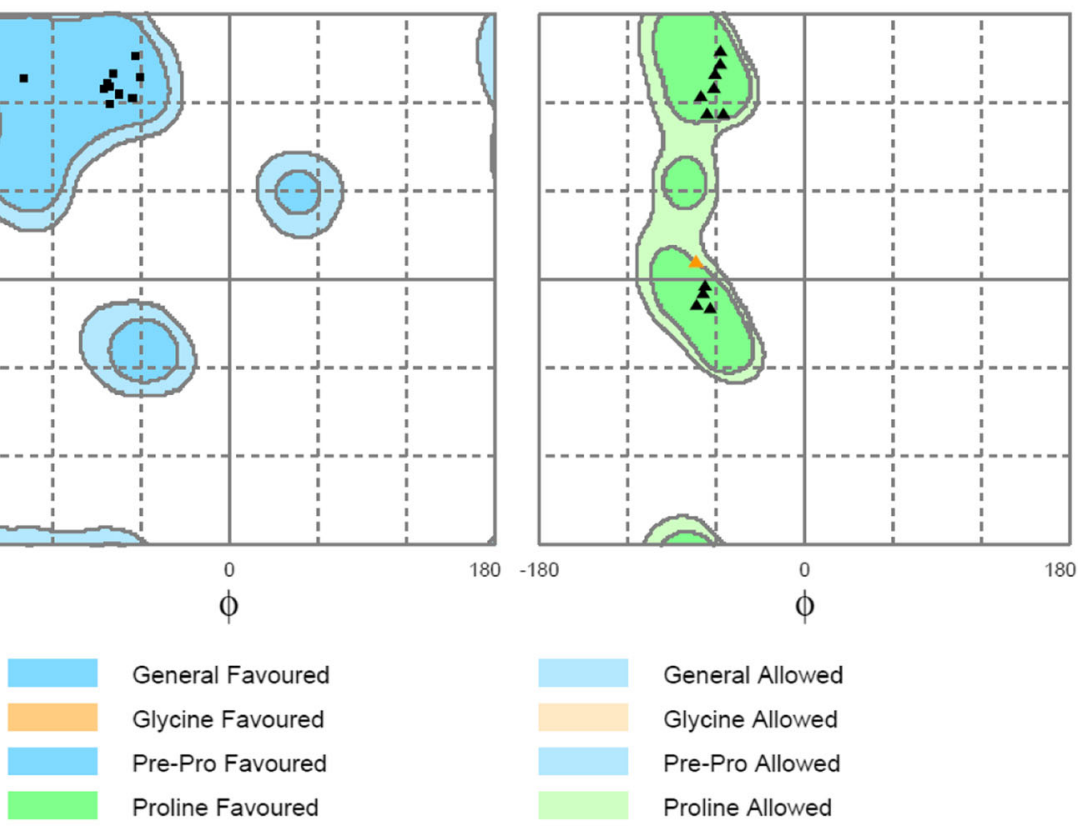

General Favoured

Glycine Favoured

Pre-Pro Favoured

Proline Favoured

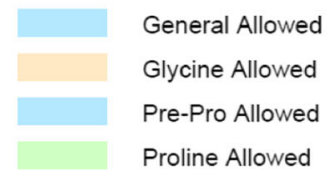

Number of residues in favoured region ( $~ 98.0 \%$ expected)

Number of residues in allowed region $(\sim 2.0 \%$ expected $)$

Number of residues in outlier region

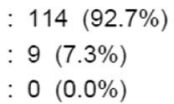

Fig. 5 Ramachandran plot statistics of BBS model

\section{Amino acid sequence analysis}

The amino acid sequence obtained from the longest ORF of BBS sequence was 193 amino acid in length and had $97 \%$ similarity and $95.2 \%$ identity over the full length of PsaD subunit of Pisum sativum photosynthesis complex I (Fig. 2).

The BBR protein sequence had a $86.2 \%$ identity and $89.1 \%$ similarity with the PsaD subunit of $P$. sativum. Both the nucleotide and protein sequence comparison clearly showed a frame shift mutation (single nucleotide deletion) leading to truncated $\mathrm{C}$ terminal caused by the single nucleotide deletion of $\mathrm{BBR}$ at $552 \mathrm{bp}$ as shown in the sequence alignments in Fig. 3. The search was repeated against PDB database and the crystal structure of $P$. sativum photosynthesis complex I was retrieved for homology modeling.

\section{Homology modeling and structure comparison}

After homology modeling and energy minimization superimposed model of the BBS sequence retained the same fold and domain structures as the PsaD subunit of 

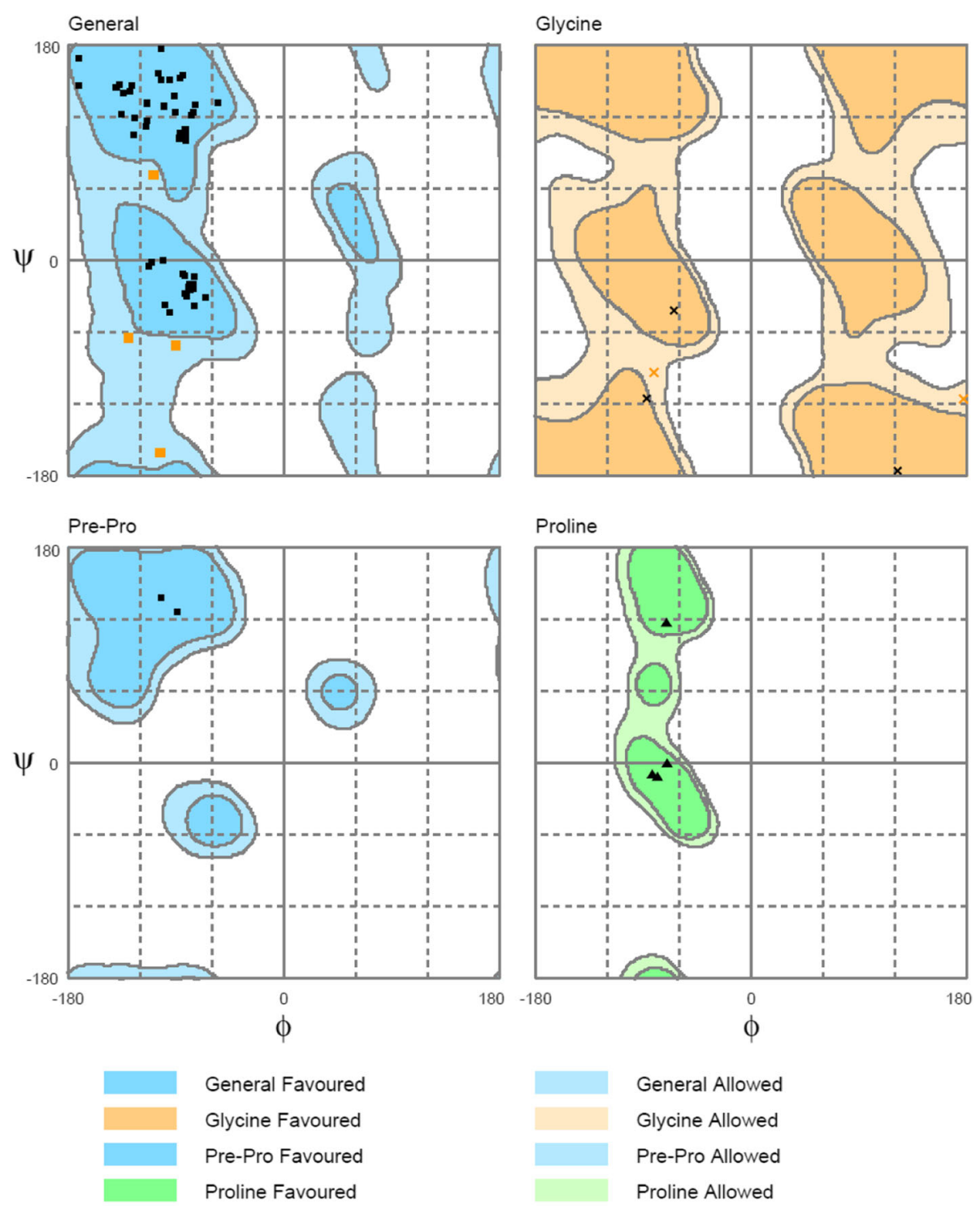

General Allowed

Glycine Allowed

Pre-Pro Allowed

Proline Allowed

$\begin{array}{ll}\text { Number of residues in favoured region ( 98.0\% expected) } & : 57(90.5 \%) \\ \text { Number of residues in allowed region }(\sim 2.0 \% \text { expected }) & : 6(9.5 \%) \\ \text { Number of residues in outlier region } & : 0(0.0 \%)\end{array}$

Fig. 6 Ramachandran plot statistics of BBR model

\begin{tabular}{|c|c|c|c|c|}
\hline VERIFY & ERRAT & PROVE & PROGHECK & WHATCHECK \\
\hline $\begin{array}{c}100.00 \% \text { of the residues have } \\
\text { averaged } 3 \mathrm{D}-1 \mathrm{D} \text { score }>=0.2 \\
\text { Pass }\end{array}$ & $\begin{array}{l}\text { Overall Quality Factor } \\
\text { A: } 79.4118\end{array}$ & $\begin{array}{l}\text { Buried outlier protein atoms total } \\
\text { from } 1 \text { Model: } 5.3 \% \text { ERROR }\end{array}$ & $\begin{array}{l}\text { Out of } 9 \text { evaluations } \\
\text { - Errors: } 5 \\
\text { - Warning: } 3 \\
\text { Pass: } 1\end{array}$ & \begin{tabular}{c|c|c|c|c|c|c|c}
1 & 2 & 3 & 4 & 5 & 6 & 7 & 8 \\
9 & 10 & 11 & 12 & 13 & 14 & 15 & 16 \\
17 & 18 & 19 & 20 & 21 & 22 & 23 & 24 \\
25 & 26 & 27 & 28 & 29 & 30 & 31 & 32 \\
33 & 34 & 35 & 36 & 37 & 38 & 39 & 40 \\
41 & 42 & 43 & 44 & 45 & 46 & 47
\end{tabular} \\
\hline
\end{tabular}

Fig. 7 Summary of BBS model quality analysis by VERIFY, ERRAT, PROVE, PROCHECK, and WHATCHECK servers 


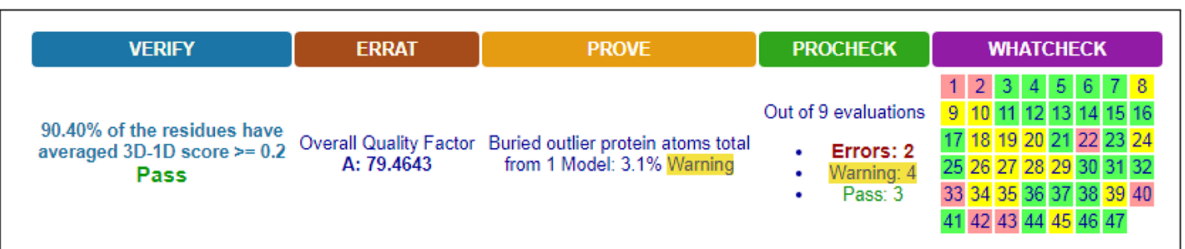

Fig. 8 Summary of BBR model quality analysis by VERIFY, ERRAT, PROVE, PROCHECK, and WHATCHECK servers

P. sativum with a RMSD value of $0.530 \AA$. More importantly, all amino acids that form $\mathrm{H}$ bonds with other subunits of the complex were conserved in P. sativum and C. sinensis (Fig. 4a). The model generated from BBR sequence clearly showed truncation of the $\mathrm{C}$ terminus, completely eliminating the anti-parallel beta strands and the $\mathrm{C}$ terminal unstructured domain (Fig. 4b, c). Rest of the structure was the same as the BBS and had an RMSD value of $0.616 \AA$ when compared to aligning region of PsaD of P. sativum.

\section{Structure validation}

After energy minimization, the models generated for BBS and BBR were showed good overall stereochemical quality as expected for modeled with high sequence identity with the template [37-39]. The BBS model had no residues in the outlier region, while $92.7 \%$ of residues lied in the favored region (Fig. 5), and BBR had 90\% of its residues in the favored region, while no residues were found in the outlier region (Fig. 6).

Further quality analysis using VERIFY3D, ERRAT, PROVE, PROCHECK, AND WHATCHECK servers indicated a good overall quality of both BBS (Fig. 7) and BBR (Fig. 8) homology models.

Generated structures and the PsaD subunit of P. sativum were further compared with physicochemical parameters such as theoretical isoelectric point (pi), estimated half-life, instability index, aliphatic index, and the grand average hydropathicity (GRAVY) of BBR, BBS, and PsaD subunit of P. sativum as given in Table 1.

\section{Molecular docking and interaction analysis}

For comparative analysis, docked complexes were compared with the interactions of the PSI complex of Pisum

Table 1 Physiological parameters of PsaD subunits

\begin{tabular}{llll}
\hline Parameter & P. sativum & BBR & BBS \\
Estimated half-life & $30 \mathrm{H}$ & $30 \mathrm{H}$ & $30 \mathrm{H}$ \\
Theoretical pl & 9.46 & 10.10 & 9.91 \\
Instability index & 47.74 & 60.65 & 45.55 \\
Aliphatic index & 72.87 & 69.04 & 69.17 \\
GRAVY & -0.513 & -0.310 & -0.419 \\
\hline
\end{tabular}

sativum (Fig. 9). Interaction analysis showed that in both $P$. sativam and BBS PsaD s, all residues that involve in $\mathrm{H}$ bonding with PsaA, Psa $\mathrm{C}$, and Psa $\mathrm{L}$ to be conserved and showing similar interaction patterns. However, BBR proteins possessed a $\mathrm{C}$ terminal truncation which prevents $\mathrm{PsaD}$ from interacting with $\mathrm{PsaC}$.

\section{Discussion}

The EST SSR 073 motif containing cDNA sequences of both TRI 2043 and TRI 2023 display significant similarity with PsaD I subunit nucleotide sequence of Chinese tea cultivar shuchazao by confirming the reliability of DNA sequence data were used in the study. Furthermore, the DNA sequences displayed high similarity with the Diospyros kaki photosystem I subunit D-I. Accordingly, both of the sequences were identified as putative PsaD subunit of photosystem I of Camellia sinensis. In almost all plants, the Psad I gene exists as a single-copy gene [40].

The CT extension of BBR does not change the sequence and the structure of protein. However, it may be involved in the post-transcriptional regulation of PsaD I expression. In fact, 5'UTR of most of the mRNA sequences contains regulatory structures such as hairpins [41]. Furthermore, the BBR sequence possesses a single nucleotide deletion at $552 \mathrm{bp}$ (deletion of $\mathrm{C}$ ) which leads to a truncation of ORF and the formation of a shorter protein product.

Homology modeling of the resulted amino acid sequences from BBS sequences using PsaD I of P. sativum as a template produced a three-dimensional structure of the protein which is very similar to the template used. PsaD I subunit is reported to be expressed as unfolded protein having a leader sequence and later undergoing proper folding when complexed with the rest of the subunit of photosystem I super complex. Also, PsaD subunits possess $\mathrm{N}$ terminal and $\mathrm{C}$ terminal domains having no rigid structure. However, these unstructured domains of PsaD I, particularly the $\mathrm{C}$ terminal unstructured domain has reported to be involved in forming $\mathrm{H}$ bonds with PsaC subunit of the complex. Therefore, energy minimization of the models generated for PsaD subunits of $\mathrm{BBS}$ and $\mathrm{BBR}$ sequences were carried out within the 


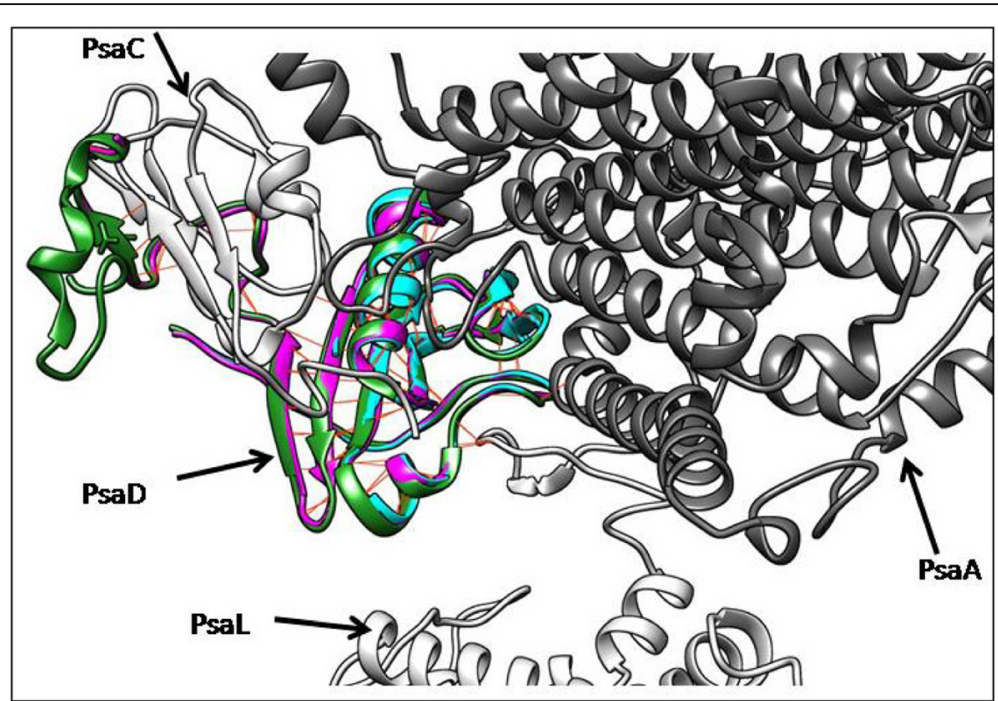

Fig. 9 Superposition of docking poses of PsaD subunits form BBS, (magenta), BBR (cyan), and P. sativum (green). Brown color lines show H bonds.

binding surface of PsaD I complex with the PsaD subunit.

After energy minimization, the 3D model obtained for BBS was almost identical to the secondary and tertiary structure of the PsaD I of P. sativum. Both of the generated models for BBS protein sequence and the PsaD I of $P$. sativum showed identical hydrogen bonding pattern with the other subunits of photosystem I super complex. When both models of BBS were docked against PsaD binding site of the photosynthesis complex, interestingly both protein models retained the same $\mathrm{H}$ bonding pattern within the complex by further confirming the adoption of the Psad I topology by them. Photosystem subunit D of photosystem I complex is hydrophobic and is exposed on stromal face of the thylakoid. The subunit interacts with ferredoxin in both cyanobacteria and eukaryotes [42].

The model generated for BBR had a $\mathrm{C}$ terminal truncation that eliminates the entire unstructured $\mathrm{C}$ terminal domain along with the $\mathrm{C}$ terminal anti-parallel beta sheets. It is unlikely that the BBR sequence would produce the functional protein because the interactions with $\mathrm{PSaC}$ is critical to maintain the stability of photosystem complex I. However, the $\mathrm{N}$ terminal extension of PsaD I in higher plants stabilizes the interactions with $\mathrm{PsaC}$ and rest of the photosystem I complex. Cross-linking study of barely, suggested that PsaD is stabilized with interaction between photosystem I H subunit [43] and Psa D is not tightly bound with photosystem I core [44]. Therefore, the $\mathrm{C}$ terminal truncation of the BBR may not involve in changing the stability of photosystem I complex and its main functions. Further, stability study of PsaD in Synechocystis has shown the reduced flavodoxin in, photosystem complex I without the PsaD subunit [45]. The mutation of BBR may be involved in so far unreported function and the predicted model may lead to discover the functions of mutated PsaD subunit.

The half-life of protein is the time it takes for half of the amount of protein in a cell to disappear after its synthesis in the cell. In this study, the half-life of all the proteins was $30 \mathrm{~h}$. The instability index provides an estimate of the stability of the protein in a test tube. A protein whose instability index is smaller than 40 is predicted as stable, while a value above 40 predicts that the protein will be unstable [46]. The results from this study recorded instability index higher than 40 in all the three proteins ( $P$. sativum, BBR, and BBS) indicating unstable properties. The aliphatic index of a protein is defined as the relative volume occupied by aliphatic side chains (alanine, valine, isoleucine, and leucine). If the aliphatic index is higher, the thermostability increases; therefore, the predicted proteins are thermostable. Isoelectric point is the condition of a solution where the amino acid produces the same amount of positive and negative charges and the ultimate charge will be zero. Isoelectric point (pI) of the three proteins was 9.4 to 10.1 and it seemed to be basic protein. The value of GRAVY spread between -0.310 and -0.514 and lower values are suggested to have good interactions between water and protein $[47,48]$.

In silico computational approach has been applied to predict protein structures of leaf rust disease resistance, and Lr 10 coding protein was identified as more resistant against the leaf rust disease of wheat [49]. However, the Psa D subunit has not been reported as associated with disease resistance up to now. Epicatechin (EC) and epigallocatachingallate (EGCG) are involved in BB disease resistance in tea [50]. Flavonoids biosynthesis 
pathway which synthesizes EC and EGCG is light sensitive and therefore, the allele may indirectly involve in the $\mathrm{BB}$ disease resistance.

\section{Conclusions}

The EST SSR 073 motif flanking sequences of Camellia sinensis is conserved in the PsaD I subunit of photosystem I complex, and the developed in silico structures of homology proteins are reliable with their physicochemical parameters. When compared with BBS, CT repeat extension of BBR did not change the topology of PsaD I subunit but the single nucleotide deletion leads to $C$ terminal truncation of BBR coding PsaD I subunit by preventing hydrogen bond interaction with other complexes of photosystem I. It can be recommended that more sequence data of EST SSR 073 motif flanking sequences in different tea cultivars and analyzing the protein model would lead to unravel the mechanism of $\mathrm{BB}$ resistance.

\section{Abbreviations}

BBS: Blister blight susceptible DNA sequence of TRI 2023; BBR: Blister blight resistance DNA sequence of TRI 2043; BB: Blister blight; SSR: Simple sequence repeat; DNA: Deoxyribonucleic acid; EST: Expressed sequence tag; PSaDI: Photosystem I subunit D protein; AER: Agro-ecological region; MRL: Maximum residual level; MAS: Marker-assisted selection; BSA: Bulk segregation analysis; NMR: Nuclear magnetic resonance; MMP: Matrix metalloproteinase; ORF: Open reading frame; NCBI: National Center for Biotechnology Information; PDB: Protein Data Bank; GRAVY: Grand average of hydropathicity; mRNA: Messenger ribonucleic acid; UTR: Untranslated region; EC: Epicatechin; EGCG: Epigallocatechingallate

\section{Acknowledgements}

Authors appreciate Tea Research Institute of Sri Lanka (TRISL) for their supporting to receive the grant. Authors thank the emeritus Professor EH Karunanayake and Professor Kamani H Tennekoon for their guidance.

\section{Authors' contributions}

KHTK and KMM conceived and designed the experiments. NHKS and KHTK analyzed the data. KHTK and KMM performed the experiment. KHTK and NHKS wrote the manuscript. SACN and KMM reviewed and revised the manuscript. OVDSJ and KMM supervised and validated the data. All authors have read and approved the manuscript.

\section{Funding}

This work was financially supported by the National Research Council, Sri Lanka (Grant No: NRC 09-066). The funding body did not participate in the design of the study, nor in the collection, analysis, and interpretation of data or in writing the manuscript.

\section{Availability of data and materials}

Authors declare that all generated and analyzed data are included in the article.

\section{Ethics approval and consent to participate}

Not applicable

\section{Consent for publication}

Not applicable

\section{Competing interests}

The authors declare that they have no competing interests.

\section{Author details}

${ }^{1}$ Institute of Biochemistry, Molecular Biology and Biotechnology, University of Colombo, Colombo, Sri Lanka. ${ }^{2}$ Current address: Department of biosystems
Technology, Faculty of Technology, University of Ruhuna, Matara, Sri Lanka. ${ }^{3}$ Department of Biotechnology, Faculty of Agriculture and Plantation Management, Wayamba University of Sri Lanka, Makandura, Gonawila, Sri Lanka. ${ }^{4}$ Department of Agricultural Biology, Faculty of Agriculture, University of Peradeniya, Peradeniya 20400, Sri Lanka.

Received: 16 March 2020 Accepted: 4 June 2020

Published online: 19 July 2020

\section{References}

1. Anon (2017) Annual ITC Bulletin of Statistics. International Tea Committee. pp 51-61

2. Agnihothrudu V, Moulli BC (1991) Blister blight of tea, its control and future lines of research. In: Proceedings of International Symposium on Tea Science, Shizuoka, Japan. 26-29 Aug 1991. p. 655-59

3. TRISL.(2002) TRI advisory Circular, no DM: 1 - protection of tea from blister blight.Talawakelle;TRISL. 1-5

4. Baby UI, Balasubramanian S, Ajay D, Premkumar R (2004) Effect of ergosterol biosynthesis inhibitors on blister blight disease, the tea plant and quality of made tea. Crop Protection. 23:795-800

5. Barooahi AK, Kalita N, Borthakur MN, Barooaht A, Barman T (2002) Non-tariff trade barriers - pesticide residues and heavy metals in tea and approaches to mitigate them. Two and a Bud. 59(2):1-8

6. Karunarathna KHT (2019) Use of SSR markers for genetic characterization and finger-printing of recommended tea (Camellia sinensis L) cultivars and identification of potential marker/s for blister blight disease resistance. Dissertation. Institute of Biochemistry, Molecular Biology and Biotechnology (IBMBB), University of Colombo

7. Canduri F, Jr WDA (2008) Protein Crystallography in Drug Discovery. Current Drug Targets 9(12):1048-1053. https://doi.org/10.2174/ 138945008786949423

8. Gupta CL, Akhtar S, Bajpai P (2014) In silico protein modeling: possibilities and limitations. EXCLI J.13:513-5. https://www.ncbi.nlm.nih.gov/pmc/ articles/PMC4467082/

9. Fadel V, Bettendorff $P$, Herrmann $T$, Jr WFDA, Oliveira EB, Yamane $T$ Wüthrich K (2005) Automated NMR structure determination and disulfide bond identification of the myotoxin crotamine from Crotalus durissus terrificus. Toxicon 46(7):759-767. https://doi.org/10.1016/j. toxicon.2005.07.018

10. Fiser A (2010) Template-based protein structure modeling. Methods Mol Biol 673:73-94. https://doi.org/10.1007/978-1-60761-842-3 6

11. Bitencourt-Ferreira G, Azeved WFD (2019) Homology Modeling of Protein Targets with MODELLER. Methods in Molecular Biology Docking Screens for Drug Discovery. pp. 231-249. https://doi.org/10.1007/978-1-4939-9752-7_15

12. Azevedo WFD, Canduri F, Oliveira JSD, Basso LA, Palma MS, Pereira JH, Santos DS (2002) Molecular model of shikimate kinase from Mycobacterium tuberculosis. Biochemical and Biophysical Research Communications 295(1): 142-148. https://doi.org/10.1016/s0006-291x(02)00632-0

13. Pereira JH, Canduri F, Oliveira JSD, Silveira NJFD, Basso LA, Palma MS, Santos DS (2003) Structural bioinformatics study of EPSP synthase from Mycobacterium tuberculosis. Biochemical and Biophysical Research Communications 312(3):608-614. https://doi.org/10.1016/j.bbrc.2003.10.175

14. Joshi YN, Gajul SG (2019) In-silico Homology Modeling of MMP25 involved in Asthma. IJSRST.4:9: 202-208

15. Satyanarayana SDV, Krishna MSR, Kumar PP, Jeereddy S (2018) In silico structural homology modeling of nif A protein of rhizobial strains in selective legume. plantsJournal of Genetic Engineering and Biotechnology 16:731-737

16. Mistry P, Kalaria RK, Patel Al (2019) Molecular identification and in silico characterization of coat protein in chilli leaf curl virus associated in chilli from south Gujarat region of India, International Journal of Chemical Studies 7(4):711-718

17. Aamir M, Singh VK, Dubey MK, Meena M, Kashyap SP, Katari SK, Upadhyay RS, Umamaheswari A, Singh S (2018) In silico Prediction, Characterization, Molecular Docking, and Dynamic Studies on Fungal SDRs as Novel Targets for Searching Potential Fungicides Against Fusarium Wilt in Tomato. Front. Pharmacol 9:1038, https://doi.org/10.3389/fphar.2018.01038

18. Mewan KM (2011) Application of DNA Markers for Genetic Conservation and Breeding of Tea [dissertation]. Department of Plant Sciences, Faculty of Science, University of Colombo 
19. Altschul S, Gish W, Miller W, Myers E, Lipman D (1990) Basic local alignment search tool. J. Mol. Biol 215:403-410

20. Rombel IT, Sykes KF, Rayner S, Johnston SA (2002) "ORF-FINDER: a Vector for High-Throughput Gene Identification". Gene 282(1-2):33-41. https://doi.org/ 10.1016/s0378-1119(01)00819-8

21. Altschul SF, Gish W, Miller W, Myers EW, Lipman DJ (1990) Basic local alignment search tool. J Mol Biol 215(3):403-10. https://doi.org/10.1016/ S0022-2836(05)80360-2

22. Boutet $E$, Lieberherr $D$, Tognolli $M$, Schneider $M$, Bansal $P$, Bridge AJ, et al (2016) UniProtKB/Swiss-Prot, the manually annotated section of the UniProt KnowledgeBase: how to use the entry view. Methods Mol Biol 1374:23-54. https://doi.org/10.1007/978-1-4939-3167-5_2

23. Minai L, Cohen Y, Chitnis PR, Nechushtai R (1996) Photosystem I complex in two steps. PNAS 93(13):6338-6342. https://doi.org/10.1073/pnas.93.13.6338

24. Lagoutte B, Hanley J, Bottin H (2001) Multiple Functions for the C Terminus of the PsaD subunit in the Cyanobacterial Photosystem I Complex. Plant Physiology 126:307-316

25. Fiser A, Sali A (2003) ModLoop: automated modeling of loops in protein structures. Bioinformatics application notes 19(18):2500-2501. https://doi. org/10.1093/bioinformatics/btg362

26. Mazor Y, Borovikova A, Caspy I. et al (2017) Structure of the plant photosystem I super complex at $2.6 \AA$ resolution. Nature Plants 3:17014. https://doi.org/10.1038/nplants.2017.14

27. Pettersen EF, Goddard TD, Huang CC, Couch GS, Greenblatt DM, Meng EC, et al (2004) UCSF Chimera - A visualization system for exploratory research and analysis. Journal of computational chemistry 25(13):1605-12. https://doi. org/10.1002/jcc.20084

28. Bitencourt-Ferreira G, Azevedo WFD (2019) Docking with SwissDock. Methods in Molecular Biology Docking Screens for Drug Discovery 189-202. https://doi.org/10.1007/978-1-4939-9752-7_12

29. Croll TI, Sammito MD, Kryshtafovych A, Read RJ (2019) Evaluation of template-based modeling in CASP13. Proteins 1-15

30. Cornell WD, Cieplak P, Bayly Cl, Gould IR, Merz KM, Ferguson DM et al (1995) A second generation force field for the simulation of proteins, nucleic acids, and organic molecules. J Am Chem Soc 117:5179-5197

31. Hornak V, Abel R, Okur A, Strockbine B, Roitberg A, Simmerling C (2006) Comparison of multiple Amber force fields and development of improved protein backbone parameters. Proteins 65:712-725

32. Bitencourt-Ferreira G, Veit-Acosta M, Azevedo WFD (2019) Van der Waals Potential in Protein Complexes. Methods in Molecular Biology Docking Screens for Drug Discovery 79-91. https://doi.org/10.1007/978-1-4939-9752-7_6

33. Ramachandran GN, Ramakrishnan C, Sasisekharan V (1963) Stereochemistry of polypeptide chain configurations. J Mol Biol 7:95-99

34. Vriend G (1990) WHAT IF: a molecular modeling and drug design program. J Mol Graph 8:52-56

35. Gasteiger E, Hoogland C, Gattiker A, Duvaud S, Wilkins MR, Appel RD. et al (2005) Protein identification and analysis tools on the ExPASy server. In Walker JM (ed) The Proteomics Protocols Handbook. Humana Press, Totowa, NJ, pp. 571-607

36. Zundert VGCP, Rodrigues JPGLM, Trellet M, Schmitz C, Kastritis PL, Karaca E. et al (2016) The HADDOCK 2.2 Web Server: User-Friendly Integrative Modeling of Biomolecular Complexes. J Mol Biol 428(4):720-725. https://doi. org/10.1016/j.jmb.2015.09.014

37. Vilar S, Sobarzo-Sánchez E, Uriarte E (2019) In Silico Prediction of Pglycoprotein Binding: Insights from Molecular Docking Studies. Curr Med Chem 26(10):1746-1760. https://doi.org/10.2174/0929867325666171129121924

38. Silveira NJFD, Bonalumi C E, Uchôa, HB, Pereira, JH, Canduri F, Azevedo WFD (2006) DBMODELING: A Database Applied to the Study of Protein Targets From Genome Projects. Cell Biochem Biophys 44(3):366-374. https://doi.org/10.1385/cbb:44:3:366

39. Azevedo WFD (2011) Molecular Dynamics Simulations of Protein Targets Identified in Mycobacterium tuberculosis. Curr Med Chem 18(9):1353-1366. https://doi.org/10.2174/092986711795029519

40. Minai L, Fish A, Yahana MD, Verchovsky L,nechustai R (2001) The Assembly Of The Psad Subunit Into The Membranal Photosystem I Complex Occurs via An exchange Mechanism. Biochemistry 40(43):12754-12760. https://doi. org/10.1021/bi015694i

41. Leppek K, Das R, Barna M (2017) Functional 5' UTR Mrna Structures In Eukaryotic Translation Regulation And How To Find Them. Nature Reviews Molecular Cell Biology 19(3):158-174. https://doi.org/10.1038/nrm.2017.103
42. Andersen B, Koch B, Scheller HV (1992) Structural and functional analysis of the reducing side of photosystem I. Physiol Plant 84:154-161

43. Naver H, Schott MP, Andersen B, Moller BL, Scheller HV (1995) Reconstitution of barley photosystem I reveals that the N-terminus of the PSI-D subunit is essential for tight binding of PSI-C. Physiologia Plantarum 95(1):19-26

44. Naver H, Haldrup A, Scheller HV (1999) Cosuppression of photosystem I subunit PSI-H in Arabidopsis thaliana. Efficient electron transfer and stability of photosystem I is dependent upon the PSI-H subunit. J Biol Chem 274: 10784-10789

45. Xu Q, Jung YS, Chitnis VP, Guikema JA, Golbeck JH, Chitnis PR (1995) Mutational analysis of photosystem I polypeptides in Synechocystis sp. PCC 6803. Subunit requirements for reduction of NADP+ mediated by ferredoxin and flavodoxin. J Biol Chem 269(34):21512-21518

46. Guruprasad K, Reddy BV, Pandit MW (1990) Correlation between stability of a protein and its dipeptide composition: a novel approach for predicting in vivo stability of a protein from its primary sequence. Protein Eng 4(2): 155-161. https://doi.org/10.1093/protein/4.2.155

47. Verma A, Singh VK, Gaur S (2016) Computational based functional analysis of Bacillus phytases. Comp Biol Chem 60:53-58. https://doi.org/10.1016/j. compbiolchem.2015.11.001

48. Pramanik K, Soren T, Mitra S, Maiti TK (2017) In silico structural and functional analysis of Mesorhizobium ACC deaminase. Comp Biol Chem 68: 12-21. https://doi.org/10.1016/j.compbiolchem.2017.02.005

49. Kumar A, Mishra DC, Rai A, Gajula MNVP (2013) In Silico analysis of protein protein interaction between resistance and virulence protein during lea rust disease in wheat (Triticum aestivum L.). World Research Journal of Peptide and Protein. 2. 2278-4586

50. Punyasiri PAN, Abeysinghe ISB, Kumar V (2005) Preformed and induced chemical resistance of tea leaf against Exobasidium vexans infection. J Chem Ecol 13:15-24

\section{Publisher's Note}

Springer Nature remains neutral with regard to jurisdictional claims in published maps and institutional affiliations.

\section{Submit your manuscript to a SpringerOpen ${ }^{\circ}$ journal and benefit from:}

- Convenient online submission

- Rigorous peer review

- Open access: articles freely available online

- High visibility within the field

- Retaining the copyright to your article

Submit your next manuscript at $>$ springeropen.com 